\title{
MEASURING TRANSVERSITY WITH T-ODD SINGLE PARTICLE PRODUCTION
}

\author{
A. BACCHETTA \\ Department of Theoretical Physics, Faculty of Science, Free University \\ De Boelelaan 1081, NL-1081 HV Amsterdam, The Netherlands
}

\begin{abstract}
The transversity distribution can be measured at leading-twist in single-particle inclusive DIS, where it appears in connection with chiral-odd fragmentation functions. Among these functions, we focus on the time-reversal odd ones. They occur in fragmentation to unpolarized hadrons or polarized spin-one hadrons. Using a simple model, we show how these functions can be non-vanishing and we discuss the asymmetries where they can be measured together with the transversity distribution.
\end{abstract}

The quark transversity distribution $h_{1}$ is an essential component in the description of the nucleon's spin. Being chiral-odd, $h_{1}$ must be coupled to another chiral-odd function to appear in a physical observable. In 1-particleinclusive deep inelastic scattering, it can occur in transverse spin asymmetries at leading order in $1 / Q$, in connection with a fragmentation function. When the final state hadron has no spin or spin 1 , the involved chiral-odd fragmentation functions belong to the class of the so-called time-reversal odd (T-odd) functions. We are going to focus on these.

It is well established that T-odd fragmentation functions do not vanish in the case of two-hadron production日, but it is possible to show that this happens in one-hadron production, too. In fact, the key feature to produce a T-odd function is the presence of an interference between two scattering amplitudes with different imaginary parts. In the case of two-hadron production, such an interference can occurr, for instance, between the resonant and the non-resonant channels. In the case of one-hadron production, the simplest expedient is to include one-loop corrections and to produce an interference between the tree-level and the one-loop amplitudes.

To show that this procedure indeed yelds non-zero T-odd functions, in a recent article $\mathrm{l}$ we used a simple model to calculate the Collins function $\mathrm{A}$, which is the archetype of a T-odd function. Considering the fragmentation process $q^{*}(k) \rightarrow \pi(p) X$, we define the Collins function, which depends on the longitudinal momentum fraction $z_{\pi}$ of the pion and the transverse momentum $k_{T}$ of the quark, as

$$
\frac{\epsilon_{T}^{i j} k_{T j}}{M_{\pi}} H_{1}^{\perp}\left(z_{\pi}, k_{T}^{2}\right)=\left.\frac{1}{4 z_{\pi}} \int \mathrm{d} k^{+} \operatorname{Tr}\left[\Delta(k, p) \mathrm{i} \sigma^{i-} \gamma_{5}\right]\right|_{k^{-}=\frac{p^{-}}{z_{\pi}}},
$$




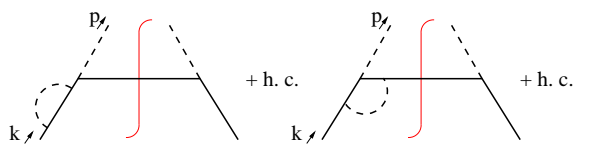

(a)

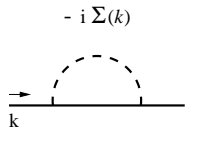

(b)

Figure 1. One-loop corrections to the fragmentation of a quark into a pion relevant for the calculation of the Collins function (a), and details of the self-energy and vertex corrections (b).

with $M_{\pi}$ denoting the pion mass, $\epsilon_{T}^{i j} \equiv \epsilon^{i j-+}$ and $\Delta(k, p)$ denoting the quarkquark light-front correlation function

$$
\Delta(k, p)=\sum_{X} \int \frac{\mathrm{d}^{4} \xi}{(2 \pi)^{4}} \mathrm{e}^{+\mathrm{i} k \cdot \xi}\langle 0|\psi(\xi)| \pi, X\rangle\langle\pi, X|\bar{\psi}(0)| 0\rangle .
$$

To describe the matrix elements in the correlation function, we use a pseudoscalar coupling between quarks and pions given by the interaction Lagrangian $\mathcal{L}_{I}(x)=\mathrm{i} g \bar{q}(x) \gamma_{5} q(x) \pi(x)$. This is clearly an oversimplified approach, but the model contains the essential elements required for our discussion. In particular, it is time-reversal invariant.

The one-loop diagrams contributing to the Collins function are shown in Fig. 1 (a). In the computation, the relevant components to be included are the self-energy correction and the vertex correction. They are sketched in Fig. 1 1 (b). The functions $\Sigma(k)$ and $\Gamma(k, p)$ appearing in the diagrams can be parametrized as

$$
\Sigma(k)=A \not k+B m, \quad \Gamma(k, p)=C+D \not p+E \not k+F \not p .
$$

After calculating and summing the contributions of the four relevant diagrams, we insert the resulting correlation function in Eq. (11) to obtain the result

$$
\begin{aligned}
& H_{1}^{\perp}\left(z_{\pi}, k_{T}^{2}\right)= \\
& \left.\frac{g^{2} M_{\pi}}{8 \pi^{3}} \frac{1}{1-z_{\pi}}\left(\frac{m \operatorname{Im}(A+B)}{\left(k^{2}-m^{2}\right)^{2}}+\frac{\operatorname{Im}(D+E+m F)}{\left(k^{2}-m^{2}\right)}\right)\right|_{k^{2}=\frac{z_{\pi}}{1-z_{\pi}} k_{T}^{2}+\frac{m^{2}}{1-z_{\pi}}+\frac{M_{\pi}^{2}}{z_{\pi}}},
\end{aligned}
$$

where $m$ is the mass of the quark.

Thereby, we have shown that the Collins function can be generated by including one-loop corrections in the fragmentation process. Though we performed an explicit calculation using a simple model, we believe that the same 
procedure can produce T-odd fragmentation functions in a much wider class of situations, with different models and different particles in the final states. The inclusion of one-loop corrections is therefore a simple and general way to produce T-odd single particle fragmentation functions and gives a convincing indication that these functions are non-zero. Given these considerations, one-particle inclusive DIS stands out as a promising process to investigate the transversity distribution of the nucleon.

The first case where the transversity distribution can be observed with T-odd functions is the production of a pion (or any unpolarized hadron) from a transversely polarized target. To single out the contribution containing the transversity distribution, it is required to measure the azimuthal asymmetry

$$
\begin{aligned}
& \left\langle\frac{\left|P_{\pi \perp}\right|}{z_{\pi} M_{\pi}} \sin \left(\phi_{\pi}^{\ell}+\phi_{S}^{\ell}\right)\right\rangle_{T} \\
& \equiv \int \mathrm{d} \phi_{S}^{\ell} \mathrm{d} \phi_{\pi}^{\ell} \mathrm{d}\left|P_{\pi \perp}\right| \frac{\left|P_{\pi \perp}\right|^{2}}{z_{\pi} M_{\pi}} \sin \left(\phi_{\pi}^{\ell}+\phi_{S}^{\ell}\right) \frac{\mathrm{d} \sigma^{\uparrow}-\mathrm{d} \sigma^{\downarrow}}{\mathrm{d}\left(x_{B}, z_{\pi}, y, \phi_{S}^{\ell}, \phi_{\pi}^{\ell},\left|P_{\pi \perp}\right|\right)} \\
& =\frac{4 \pi \alpha^{2} s}{Q^{4}} x_{B}\left|S_{T}\right|(1-y) \sum_{a} e_{a}^{2} h_{1}^{a}\left(x_{B}\right) H_{1}^{\perp(1) a}\left(z_{\rho}\right),
\end{aligned}
$$

where $P_{\pi \perp}$ is the perpendicular component of the pion momentum, $y$ is the energy fraction of the scattered lepton, $\phi_{S}^{\ell}$ and $\phi_{\pi}^{\ell}$ are respectively the azimuthal angles of the target's transverse spin $\left(S_{T}\right)$ and of the outgoing pion, with respect to the scattering plane. The summation runs over quark flavors with charge $e_{a}$.

In the case of the production of spin-one hadrons, the extra information about the polarization of the final state is contained in the dependence of the cross section on the decay angles of the decay products. The azimuthal asymmetries can in general be defined as ${ }^{a}$

$$
\langle W\rangle_{T}=\int \mathrm{d} \phi_{S}^{\ell} \mathrm{d} \phi_{\rho}^{\ell} \mathrm{d}\left|P_{\rho \perp}\right| \mathrm{d} \phi_{\pi}^{\ell} W \frac{\mathrm{d} \sigma^{\uparrow}-\mathrm{d} \sigma^{\downarrow}}{\mathrm{d}\left(x_{B}, z_{\rho}, y, \phi_{S}^{\ell}, \phi_{\rho}^{\ell},\left|P_{\rho \perp}\right|, \phi_{\pi}^{\ell}, \theta_{\pi}\right)},
$$

where $W=W\left(\phi_{\rho}^{\ell},\left|P_{\rho \perp}\right|, \phi_{\pi}^{\ell}, \theta_{\pi}\right), \phi_{\pi}^{\ell}$ is the azimuthal angle of one of the decay pions with respect to the scattering plane and $\theta_{\pi}$ is the polar angle of the same pion as measured in the $\rho$ meson rest-frame with respect to the direction of the meson in the target rest-frame.

The following asymmetries containing the transversity distribution can

${ }^{a}$ Although in the following we specialize the discussion on the $\rho$ meson, everything can be applied also to higher mass vector mesons.

dis01proc: submitted to World Scientific on December 20, 2018 
then be obtained at leading order in $1 / Q$ :

$$
\begin{aligned}
& \left\langle\sin \left(\phi_{\pi}^{\ell}+\phi_{S}^{\ell}\right)\right\rangle_{T} \\
& =\frac{2 \pi \alpha^{2} s}{Q^{4}} x_{B}\left|S_{T}\right|(1-y)\left|\sin 2 \theta_{\pi}\right| \sum_{a} e_{a}^{2} h_{1}^{a}\left(x_{B}\right) H_{1 L T}^{a}\left(z_{\rho}\right), \\
& \left\langle\frac{\left|P_{\rho \perp}\right|}{2 z_{\rho} M_{\rho}} \sin \left(2 \phi_{\pi}^{\ell}+\phi_{S}^{\ell}-\phi_{\rho}^{\ell}\right)\right\rangle_{T} \\
& =\frac{4 \pi \alpha^{2} s}{Q^{4}} x_{B}\left|S_{T}\right|(1-y) \sin ^{2} \theta_{\pi} \sum_{a} e_{a}^{2} h_{1}^{a}\left(x_{B}\right) H_{1 T T}^{(1) a}\left(z_{\rho}\right), \\
& \left\langle\frac{\left|P_{\rho \perp}\right|}{2 z_{\rho} M_{\rho}} \sin \left(\phi_{S}^{\ell}+\phi_{\rho}^{\ell}\right)\right\rangle_{T} \\
& =\frac{4 \pi \alpha^{2} s}{Q^{4}} x_{B}\left|S_{T}\right|(1-y) \frac{\left(1-3 \cos ^{2} \theta_{\pi}\right)}{2} \sum_{a} e_{a}^{2} h_{1}^{a}\left(x_{B}\right) H_{1 L L}^{\perp(1) a}\left(z_{\rho}\right), \\
& \left\langle\frac{\left|P_{\rho \perp}\right|^{2}}{2 z_{\rho}^{2} M_{\rho}^{2}} \sin \left(\phi_{S}^{\ell}+2 \phi_{\rho}^{\ell}-\phi_{\pi}^{\ell}\right)\right\rangle_{T} \\
& =\frac{4 \pi \alpha^{2} s}{Q^{4}} x_{B}\left|S_{T}\right|(1-y)\left|\sin 2 \theta_{\pi}\right| \sum_{a} e_{a}^{2} h_{1}^{a}\left(x_{B}\right) H_{1 L T}^{\perp(2) a}\left(z_{\rho}\right) .
\end{aligned}
$$

Note that the asymmetry of Eq. (7) could represent a very convenient way to measure the transversity distribution because it does not require the perpendicular momentum of the outgoing hadron to be detected. It represents a specific case of the more general two-pion production? and should exhibit a well known Breit-Wigner dependence on the invariant mass of the pion couple.

This work has been done in collaboration with R. Kundu, A. Metz and P. Mulders at the Free University of Amsterdam.

\section{References}

1. J. P. Ralston and D. E. Soper, Nucl. Phys. B 152 (1979) 109; R. L. Jaffe and X. Ji, Phys. Rev. Lett. 67 (1991) 552; V. Barone, A. Drago and P. G. Ratcliffe, hep-ph/0104283.

2. M. Radici, these proceedings.

3. A. Bacchetta, R. Kundu, A. Metz and P. J. Mulders, Phys. Lett. B 506 (2001) 155 .

4. J. Collins, Nucl. Phys. B396 (1993) 161; D. Boer, these proceedings.

5. A. Bacchetta and P. J. Mulders, Phys. Rev. D 62 (2000) 114004. 